\title{
BACTERIAL STRUCTURE AND ANTIMICROBIAL SUSCEPTIBILITY OF MOST FREQUENT CLINICAL ISOLATES IN VARNA UNIVERSITY HOSPITAL FOR THE PERIOD 2003-2005
}

\author{
Bojkova K., T. Stoeva, V. Kaludova, V. Kamenova, V. Rusev \\ Department of microbiology and virology, Medical University - Varna
}

Reviewed by: Assoc. Prof. J. Radkov, MD, PhD

\begin{abstract}
Introduction: Registering and analyzing the etiological bacterial structure of the University Hospital is a process which together with the monitoring of the antimicrobial resistance gives helpful information to the clinicians. Objectives: The spectrum of most frequently isolated bacterial pathogens from different clinical materials is showed comparatively for the three years period 2003-2005. The recent trends of antimicrobial resistance of most clinically important pathogens in The University Hospital "St. Marina" are studied for the same period. Material and methods: A total number of 9309 clinical materials were studied, from which 23306 bacterial strains were isolated. Isolation and identification were done by conventional, automated and semi-automated methods. Antimicrobial susceptibility testing was performed, using disc-diffusion method, strictly following the NCCLS recommendations. Results and discussion: During the studied period the Gram-negative bacterial species prevail over the other species $(41 \%-53 \%)$. The percentage of Candida spp. is relatively high $(\sim 16,8 \%$ for 2005). The most commonly isolated bacterial species is E.coli $(15,69 \%-16,25 \%)$, followed by $S$. aureus (7, 4\%-16,25\%). For the three year period the problematic pathogens as isolates Enterobacteriaceae, which are producers of ESBLs, MRSA, PNSP and multidrugresistant nonfermenters show a trend for continuous increasing. Conclusion: Regular monitoring the antimicrobial susceptibility is necessary to collect data concerning the local hospital resistance, which is of great importance for adequate therapeutic strategy.
\end{abstract}

Keywords: bacterial spectrum, antimicrobial susceptibility, antibiotic policy.

With the opening of Varna University Hospital (now UMHAT "St. Marina") at the end of 1989, the Clinical Microbiology Laboratory starts its functioning. The laboratory passed through several stages of its development, till it came to the present state as a structure with important impact on the process of diagnosis and therapy of the patients. The main tasks of the laboratory are to provide information about the presence or absence of microorganisms in the clinical materials tested, which will help the clinical diagnosis; to give information about antimicrobial susceptibility of the etiological agents and to collect data, concerning the local (hospital) resistance, helping the optimization of the antimicrobial therapy.

The aim of this work is to study the spectrum of bacterial species, isolated from different clinical materials (blood, urine, sputum) and to reveal the recent trends of antimicrobial resistance in UMHAT "St. Marina" for the period 2003-2005.

Address for correspondence:

K. Bojkova, Dept. of microbiology and virology, Medical University Prof. Dr. Paraskev Stoyanov, 55 Marin Drinov St., BG-9002, Varna, BULGARI

\section{MATERIAL AND METHODS}

A total number of 28856 urine samples 11507 sputums and 10131 blood samples were tested. The isolation and identification of bacterial strains were assessed by conventional, semi-automated (Crystal/BD, Lachema/Czech Republic) and automated systems (Bactec 9120, Sceptor/BD). Disc Diffusion Method performed the antimicrobial susceptibility, strictly following the NCCLS recommendation, and E-test for some isolates.

\section{RESULTS AND DISCUSSION}

The total number of the tested clinical materials is shown on fig.1. The trend for continuous increasing the number of microbiological examinations is obvious. From one side this is a result of establishing of new departments in UMHAT "Saint Mariana", and from another - due to the increasing number of patients, suffering from infectious diseases and the necessity of microbiological examinations, as well as the growing role of the laboratory in the diagnostic and therapeutic processes. 


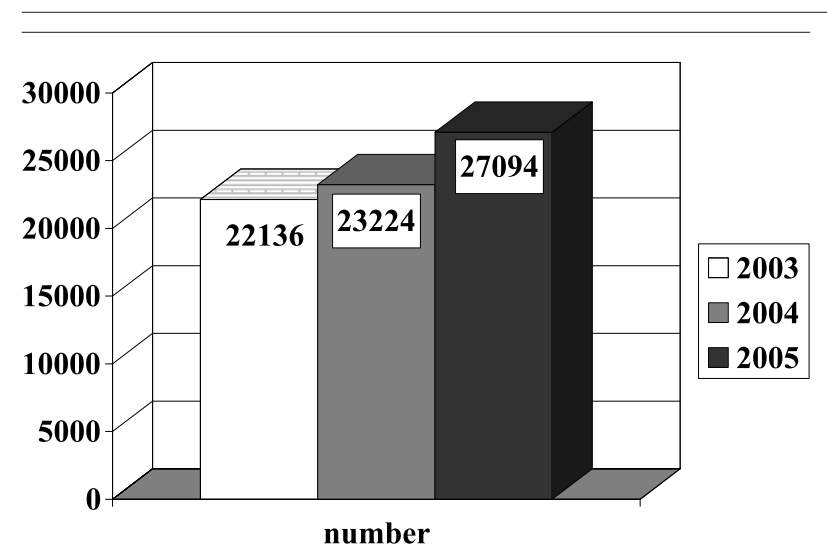

Fig. 1. A total number of tested clinical materials for the period 2003-2005.

During the three years period a trend of decreasing the number of isolates E.coli (from $17 \%$ to $15,7 \%$ during 2005 ) at the expense of the increasing number of isolates Candida spp. $(17 \% / 2003-18,4 \% / 2005)$ was found (figure 2$)$. The lower proportion of Gram-positive isolates stays stable during the studied period, which data correlates with the data from National Surveillance System, carried out for the hospital laboratory (1).

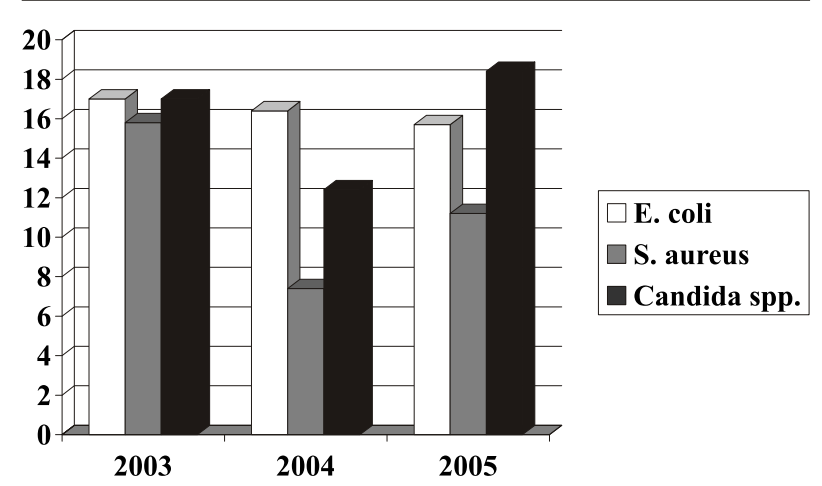

Fig. 2. Most frequent isolates for the period 2003-2005 (\%).

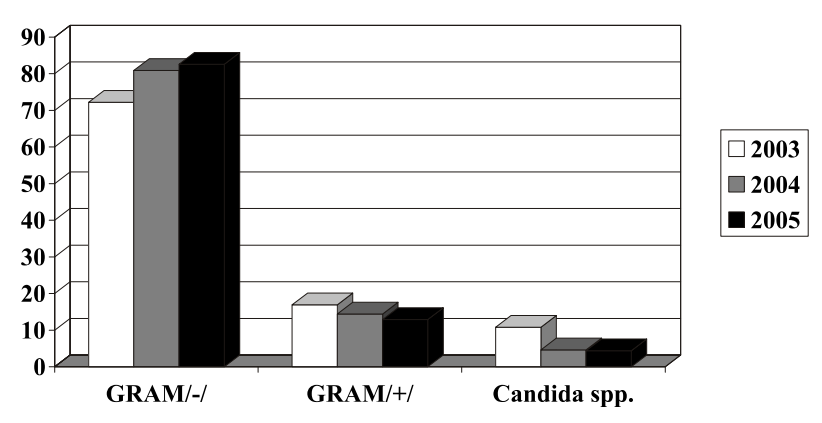

Fig. 3. Distribution of the bacterial isolates from urine samples for the period 2003-2005 (\%)

The relative rate of Gram negative isolates from urine samples (the most frequently examined clinical material) tends to increase in comparison with the Gram positive isolates and Candida spp. (figure 3).
The four most frequently isolated bacterial species from urine samples are the Gram negative E. coli, Klebsiella spp., Proteus spp. and Pseudomonas aeruginosa (figure 4).

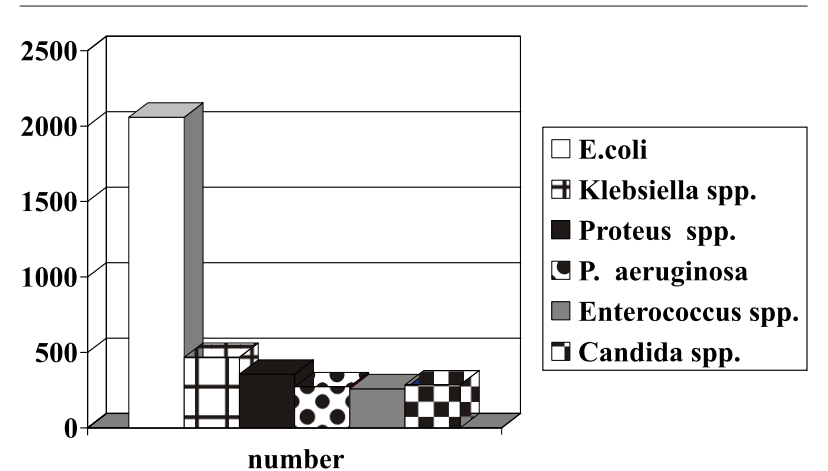

Fig. 4. Spectrum of the most frequently isolated bacterial species from urine samples for the period 2003-2005.

Blood cultures are one of the most important clinical materials for microbiological examination to diagnose bacteremia, especially in severe invasive infections. For the studied period we detect a significant increase of Gram positive bacteremias (from 51\%/2002 to $62 \% / 2005$ ) and decrease of the Gram negative and Candida spp. isolates (figure 5). Our results are similar to those, obtained from National Surveillance system for the hospital structures $(1,2)$.

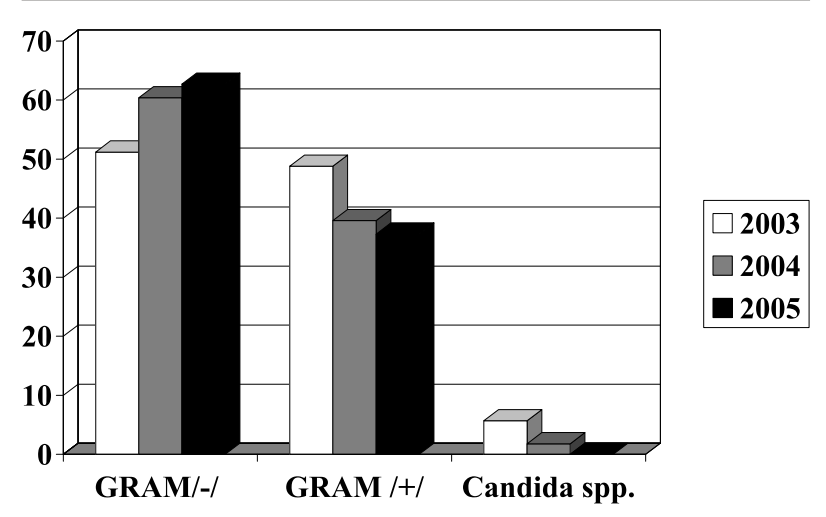

Fig. 5. Bacterial isolates from blood cultures for the period 2003-05 (\%).

CNS, E.coli and S.aureus are the bacterial species most frequently isolated from the positive blood cultures and in all most equal proportions. The explanation of this fact is difficult, especially when we take into consideration that part of the CNS isolates are contaminants, but not etiological agents and that they are isolated from single blood cultures. From the other side this may be a real increase of the etiological role of these isolates because of the increased use of intravascular devices and manipulations in our hospital. The differentiation between a clinically significant bacteremia and contamination has to be based on clinical and microbiological criteria, considered for each patient. Many authors (in Bulgaria and abroad) report similar rates of isolation of CNS from blood cultures $(1,2)$ (figure 6). S.pneumoniae is the most frequently isolated bacterial 
pathogen from sputum, followed by Gram-negative bacteria from Enterobacteriaceae, S. aureus, etc. (figure 7).

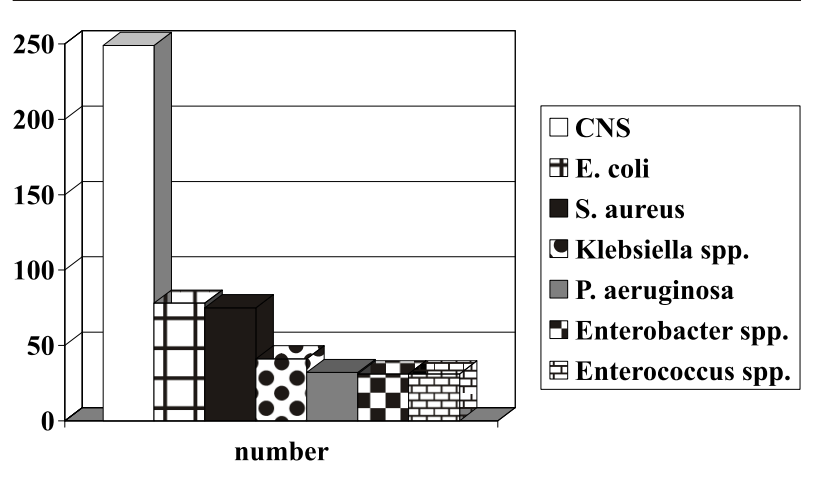

Fig. 6. Bacterial species isolated from blood cultures for the period 2003-2005.

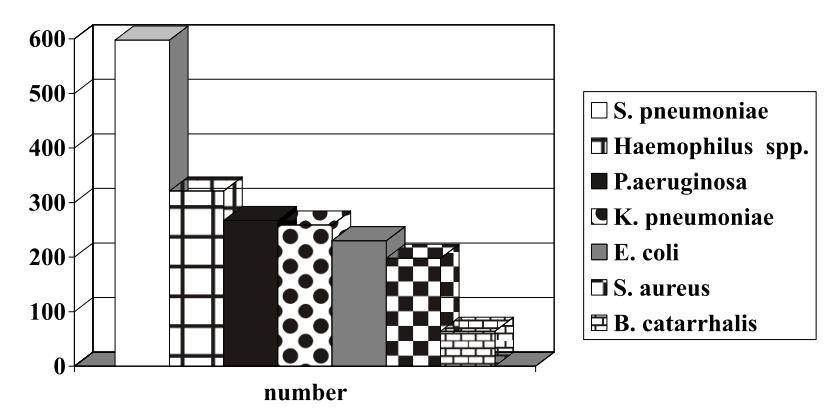

Fig. 7. Bacterial isolates from sputum for the period 2003-2005.

To optimize the therapeutic regiments the hospital bacterial resistance is monitored. Methicillin resistant S.aureus (MRSA), Methicillin resistant coagulase negative staphylococci (MRCNS), Vancomycin resistant enterococci (VRE), Penicillin nonsusceptible S.pneumoniae (PNSSP), ?nterobacteriaceae producers of expended spectrum beta-lactamases (ESBLs), carbapenem resistant NFB and ?nterobacteriaceae are poliresistant microorganisms and hence problematic.

The percentage of MRSA for the different clinical materials is as follows: lower respiratory tract: $16,8 \%-37,3 \%$ blood cultures: $0 \%-14,8 \%$. The percent of MRSA for 2005 in our hospital ranges to $16,9$.

No resistant to glycopeptide and oxazolidinones Staphylococcus spp. was detected for the studied period.

Increasing resistance to Ampicillin ( $0 \%$ to $20 \%$ ) was found for Enterococcus spp. isolates, but only for blood cultures isolates. No resistance to glycopeptide and oxazolidinones in Enterococcus spp. was detected.

Resistance to penicillin in S. pneumoniae is calculated as a sum of the resistant and intermediate isolates, so called ?enicillin non-susceptible S.pneumoniae. The rate of PNSSP for the period 2003-2005 shows a trend of slightly increase: $38,4-40 \%$.

H. Influenzae is the second most frequent isolate from sputum. Its resistance to ?rythromycin and tetracycline in- creases. Good choice for treating infections, cause by $H$. Influenzae is Amoxicillin/Clavulanic acid, because only $2 \%$ of the isolates are resistant. Low rate of resistance to second generation cephalosporins was detected $(6 \%)$. The susceptibility to third generation cephalosporins is preserved.

The isolates from ?nterobacteriaceae, that produce ESBLs, need special attention because they are resistant to all beta-lactams except carbapenems. Among the starins E.coli, isolated from blood cultures the rate of ESBLs producers varies between $9.5 \% / 2002$ to $14.3 \% / 2005$. ?SBLs $K$. pneumoniae isolates seem to be more common, a fact proved by other authors too (1). The ESBLs producers among the isolates from blood cultures and urine samples are increasing during the three year period: from $27,2 \% / 2002$ to $66,6 \% / 2005$ and from $10.5 \% / 2002$ to $45 \% / 2005$, respectively.

For the studied period no carbapenems resistant strains from Enterobacteriaceae were detected.

P.aeruginosa is one of the most important nosocomial pathogens, causing severe infections (especially in immunocompromised hosts). A recent trend of increasing the number of these isolates from blood cultures $(5,7 \% / 2002$ to $10,7 \% / 2005)$, and urine samples $(5,9 \% / 2002$ to $10,4 \% / 2005)$ was detected. The carbapenem resistance in P.aeruginosa increases from $6,5 \%$ to $17 \%$.

\section{CONCLUSIONS}

Gram-negative bacteria keep their predominant place among the whole group of bacterial isolates. The most frequently isolated species is E.coli, followed by S.aureus. Significant rate of isolation for Candida spp. was detected. Clinically significant P.aeruginosa isolates from various materials (for example urine) are increasing. Multiresistant isolates such as MRSA, ESBL producers, PNSSP show a trend to increase too. A worrisome fact is the proved high percent of ESBLs producers in different clinical materials (esp. urine), which is a result of increased antibiotic consumption. The susceptibility to strategic groups of antimicrobials (glicopeptides, oxazolidinones and carpapenems) is still preserved.

\section{REFERENCES}

1. Велинов Ц., М. Петров, А. Бъчварова, Т. Кантарджиев. Етиологична структура и резистентност на най-честите причинители на инфекции в България-BULSTAR 2004. Инфектология,v. XLIII, 2006, 8-11.

2. Mirovic, V. The frequency of isolation and resistance patterns of microorganisms from 5637 blood cultures. Clin Microboil. Infection, v.8 Suppl. 1, 2002, P928. 